\title{
Effect of Removing Atmospheric Carbon Dioxide from Eluent in Determination of Trace Level Carbonate-Carbon by Ion Exclusion Chromatography
}

\author{
Kazuichi Hayakawa, Kyoko Nomura and Motoichi Miyazaki
}

Faculty of Pharmaceutical Sciences, Kanazawa University, Takara-machi, Kanazawa 920, Japan

Keywords Carbonate-carbon, carbon dioxide, nitrogen bubbling, ion exclusion chromatography

Carbon dioxide causes atmospheric temperature increases on a global scale. On the other hand, carbon dioxide plays important roles in biological fluids such as carbonic acid, hydrogencarbonate and carbonate. In these fields, the determination of carbonate-carbon is important. Ion exclusion chromatography (IEC) ${ }^{1}$ with conductivity detection has been used mainly for the determination of carboxylic acids. Although a carbonate peak is often observed using this method, this peak has not been used in the determination of carbonate due to its low sensitivity and the narrow straight range of the calibration curve. ${ }^{2}$ Atmospheric carbon dioxide dissolved in the eluent was considered as a cause of this problem. In order to increase the sensitivity for carbonate, the use of a precolumn (anion exchanger) in combination with other two postcolumns (cation and anion exchangers) has been proposed. ${ }^{3}$ The former column removed carbon dioxide from the eluent and the latter columns enhanced the peak by converting carbonic acid to potassium hydroxide after the separation column. This system, however, is very complex and the three extra columns must be changed when exhausted.

Indirect photometric ion chromatography (PIC) is a useful method for determining ionic compounds. ${ }^{4}$ Although atmospheric carbon dioxide dissolved in the eluent interfered with the sensitive determination of hydrogen carbonate, we found that this interfering carbon dioxide could be effectively removed by bubbling nitrogen gas through the eluent. By attaching this nitrogen bubbling system to a conventional PIC system, hydrogen carbonate was determined sensitively and simultaneously with strong acidic anions. ${ }^{5}$

According to the equation,

$$
\begin{aligned}
& \mathrm{H}_{2} \mathrm{CO}_{3} \stackrel{K_{\mathrm{a} 1}}{\rightleftharpoons} \mathrm{H}^{+}+\mathrm{HCO}_{3}{ }^{-} \stackrel{K_{\mathrm{a} 2}}{\rightleftharpoons} 2 \mathrm{H}^{+}+\mathrm{CO}_{3}{ }^{2-} \\
& \quad\left(\mathrm{p} K_{\mathrm{a} 1}=6.34, \mathrm{p} K_{\mathrm{a} 2}=10.25\right),
\end{aligned}
$$

the ratio of $\mathrm{H}_{2} \mathrm{CO}_{3}$, which is calculated as aqueous carbon dioxide, is increased by decreasing the $\mathrm{pH}$. The fact that diluted acid solutions are often used as eluents for IEC suggested the easy removal of carbon dioxide from these solutions. When the nitrogen bubbling system was attached to the eluent reservoir for IEC, the interfering carbon dioxide was effectively removed. The purpose of this report is to describe the usefulness of this system in the sensitive and simultaneous determination of carbonate and carboxylates by IEC.

\section{Experimental}

\section{Chemicals and solutions}

Chemicals used were all commercially available analytical-reagent grade. Distilled and deionized water was purified again with a Millipore (Bedford, MA, USA) Milli-Q system before use. Standard solutions of carbonate, formate, acetate, propionate and $n$-butyrate were prepared according to our previous report ${ }^{5}$ as follows: stock solutions of sodium salts of hydrogencarbonate and the four carboxylates (each $0.1 \mathrm{M}$ ) were prepared with water in silicone rubber-stoppered glass vials and stored at $4^{\circ} \mathrm{C}$. After each vial, containing a small stirring bar, was filled with water under a nitrogen atmosphere and immediately stoppered with a septum, stock solutions were injected into the vial through the septum using a microsyringe. Then the mixture was stirred for $2 \mathrm{~min}$. The eluent was $1.0 \times 10^{-5} \mathrm{M}$ phosphoric acid and filtered with a membrane $(0.40 \mu \mathrm{m})$ before use.

\section{Apparatus and conditions}

The IEC system consisted of a Shimadzu (Kyoto, Japan) LP-6A pump, a Rheodyne (Cotati, CA, USA) 7125 injector with a loop of $20 \mu \mathrm{l}$, a Shimadzu CDD-6A conductivity detector and a Shimadzu C-R3A integrator. The analytical column (stainless steel, $500 \times 4.6 \mathrm{~mm}$ i.d.) was packed with a Mitsubishi Chemicals (Tokyo, Japan) MCI CK 10S (polystyrene-divinylbenzene copolymer, 
$10-12 \mu \mathrm{m}$, with a cation exchange capacity of over $2 \mathrm{meq} / \mathrm{ml}$ ). Both the column and the detection cell was set in a Shimadzu CTO-6AS column oven kept at $40^{\circ} \mathrm{C}$. The eluent flow rate was set at $1.0 \mathrm{ml} / \mathrm{min}$.

According to our previous report ${ }^{5}$, the nitrogen bubbling system consisted of a nitrogen gas bomb (purity over $99.99 \%$ ), a gas washing bottle and an eluent reservoir. The nitrogen gas was continuously bubbled through the eluent at a flow rate of $50 \mathrm{ml} / \mathrm{min}$. Other conditions were the same as in our previous report. ${ }^{5}$

\section{Results and Discussion}

In order to obtain as large a signal-to-noise ratio for carbonate, phosphoric acid, nitric acid and hydrochloric acid as possible, various concentrations between $1.0 \times 10^{-2} \mathrm{M}$ and $1.0 \times 10^{-5} \mathrm{M}$ were compared as eluents. The largest signal-to-noise ratio observed was for $1.0 \times 10^{-5} \mathrm{M}$ phosphoric acid. The aqueous $\mathrm{pH}$ of phosphoric acid, which affects the dissociation of carbonic acid, is highest at the same concentration, since $\mathrm{p} K_{\mathrm{a}}$ values of phosphoric acid $\left(\mathrm{p} K_{\mathrm{a} 1}=2.10, \mathrm{p} K_{\mathrm{a} 2}=6.71\right.$, $\mathrm{p} K_{\mathrm{a} 3}=11.8$ ) are larger than those of the other acids. Additionally, the limiting ionic equivalent of phosphate $\left(36.0 \mathrm{~S} / \mathrm{cm}^{2}\right)$ is smaller than the others. These two characteristics of phosphoric acid might be considered as reasons for the above result. Although, a small increase of peak area was observed by decreasing the concentration below $1.0 \times 10^{-5} \mathrm{M}$, the number of theoretical plates became worse. From this result, $1.0 \times 10^{-5} \mathrm{M}$ phosphoric acid was used in the following experiments. Utilizing this eluent at the flow rate of $1.0 \mathrm{ml} / \mathrm{min}$, carbonate and four carboxylates were eluted in $7 \mathrm{~min}$.

By increasing both the nitrogen flow rate and the bubbling time, carbonate became detectable as a positive peak and its height was increased. When a litre-glass bottle was used as an eluent reservoir, the nitrogen flow rate and the bubbling time necessary to obtain the constant and reproducible peak height were $50 \mathrm{ml} / \mathrm{min}$ and $1 \mathrm{~h}$, respectively. Figure 1 shows the comparison of chromatograms of carbonate, formate, acetate, propionate and $n$-butyrate by IEC with/without nitrogen bubbling. The carbonate peak ( $200 \mathrm{pmol})$ was detected at $6.0 \mathrm{~min}$ when the proposed nitrogen bubbling system was used (Fig. 1A), while it was not detected at all when the system was not used (Fig. 1B).

Using the proposed system, the calculated detection limit (signal-to-noise ratio was three) of carbonate by IEC was down to $5 \mathrm{pmol}$. This method is $\mathbf{3 0}$ times more sensitive than IEC using three extra columns ${ }^{3}$ and three times more sensitive than PIC. ${ }^{5}$ The calibration curve was linear from $60 \mathrm{pmol}$ to $20 \mathrm{nmol}$, with a correlation coefficient of 0.9999 . These results are sufficient enough to determine trace levels of carbonate. Peak heights of four carboxylates in Fig. 1A were higher by $13-20 \%$ than those in Fig. 1B. This result suggests that dissociations of carboxylic acids are increased by the removal of carbon dioxide. One reason may be the

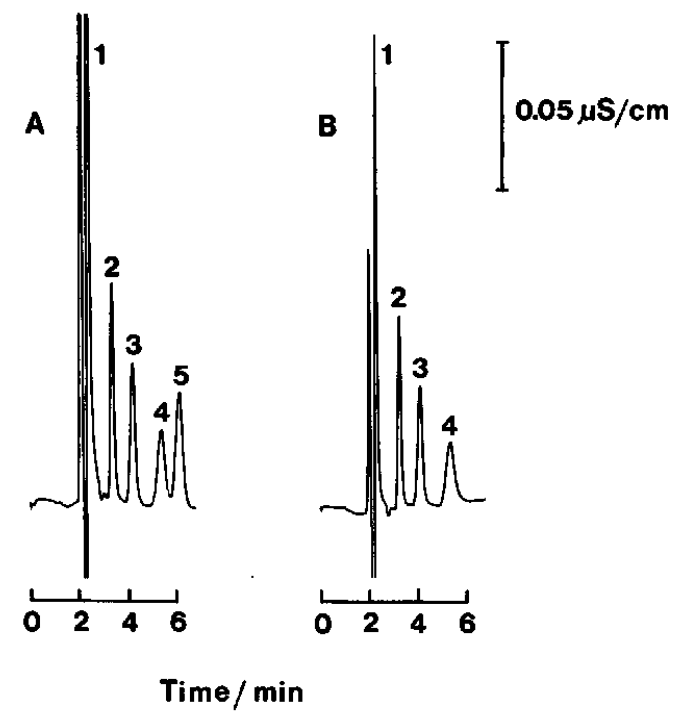

Fig. 1 Chromatograms of carbonic and carboxylic acids by IEC (A) with and (B) without nitrogen bubbling. Peaks: 1 , formate $(60 \mathrm{pmol}) ; 2$, acetate $(200 \mathrm{pmol}) ; 3$, propionate $(200$ pmol); $4, n$-butyrate (200 pmol); 5 , carbonate ( 200 pmol).

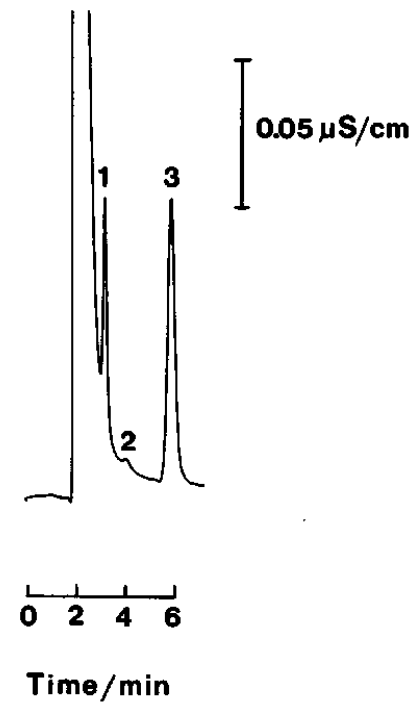

Fig. 2 Typical chromatogram of rain water. Peaks: 1, acetate $(3.3 \mu \mathrm{M}) ; 2$, propionate $(0.12 \mu \mathrm{M}) ; 3$, carbonate $(24 \mu \mathrm{M})$.

small increase in the eluent $\mathrm{pH}$.

Other advantages of this method are that the proposed IEC system does not require any extra columns and that it is easy to operate. These points might be desirable for the practical use. Figure 2 shows a typical chromatogram of rain water (Kanazawa, December 14, 1990) as an application of this method. Carbonate, acetate and propionate were simultaneously determined without any prior clean-up treatment. This method should prove useful in the simultaneous determination of carbonic and carboxylic acids in environmental and fermented samples. 
This work was partly. supported by a Grant-in-Aid for General Scientific Research No. 02670975 from the Ministry of Education, Science and Culture, Japan.

\section{References}

1. R. M. Wheaton and W. C. Bauman, Ind. Eng. Chem., 45, 228 (1953).

2. K. Tanaka and T. Ishizuka, Water Res., 16, 719 (1982).

3. K. Tanaka and J. S. Fritz, Anal. Chem., 59, 712 (1987).
4. M. Miyazaki and K. Hayakawa, "Atarashii Ion Chromatography no Tehodoki (Introduction to New Ion Chromatography, in Japanese)", Nankodo, Tokyo, 1986.

5. K. Hayakawa, S. Kitamoto, N. Okubo, S. Nakamura and M. Miyazaki, J. Chromatogr., 481, 323 (1989).

(Received September 6, 1991) (Accepted November 26, 1991) 